\title{
NEW RESULTS OF THE HIGH-GAIN HARMONIC GENERATION FREE-ELECTRON LASER EXPERIMENT*
}

\author{
A. Doyuran $^{2+}$, M. Babzien", T. Shaftan", S.G. Biedron ${ }^{\text {, }}$,L. H. Yu , I. Ben-Zvi", L.F.

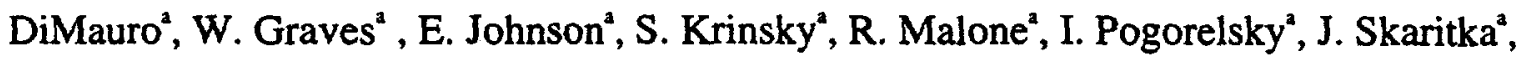 \\ G. Rakowsky", X.J. Wang", M. Woodle', V. Yakimenko', J. Jagger ${ }^{b}$, V. Sajaev ${ }^{b}$, I. \\ Vasserman ${ }^{b}$ \\ 'Brookhaven National Laboratory, Upton, New York 11973 \\ 'Advanced Photon Source, Argonne National Laboratory, Argonne, Illinois 60439
}

\section{Abstract}

We report on the experimental investigation of high-gain harmonic generation carried out at the Accelerator Test Facility at Brookhaven National Laboratory. A seed $\mathrm{CO}_{2}$ laser at a wavelength of $10.6 \mu \mathrm{m}$ was used to generate FEL output at a $5.3-\mu \mathrm{m}$ wavelength. The duration of the output pulse was measured using a second-harmonic intensity autocorrelator, and the coherence length was measured using an interferometer. We also measured the energy distribution of the electron beam after it exited the second undulator, observing behavior consistent with that is expected at saturation. The intensity of the harmonic components of the output at $2.65 \mu \mathrm{m}$ and $1.77 \mu \mathrm{m}$ were determined relative to that of the 5.3- $\mu \mathrm{m}$ fundamental. Finally, using a corrector magnet upstream of the radiator, steering effects on the trajectories of the electron and light beams were studied.

-Work supported by U.S. Department of Energy, Office of Basic Energy Sciences, under Contracts Nos. DEAC02-98CH 10886 and W-31-109-ENG-38 and by Office of Naval Research Grant no. N00014-97-1-0845. †E-mail: adoyuran $\oplus$ ic.sunysb.edu

\section{Introduction}




\section{DISCLAIMER}

This report was prepared as an account of work sponsored by an agency of the United States Government. Neither the United States Government nor any agency thereof, nor any of their employees, make any warranty, express or implied, or assumes any legal liability or responsibility for the accuracy, completeness, or usefulness of any information, apparatus, product, or process disclosed, or represents that its use would not infringe privately owned rights. Reference herein to any specific commercial product, process, or service by trade name, trademark, manufacturer, or otherwise does not necessarily constitute or imply its endorsement, recommendation, or favoring by the United States Government or any agency thereof. The views and opinions of authors expressed herein do not necessarily state or reflect those of the United States Government or any agency thereof. 


\section{DISCLAIMER}

Portions of this document may be illegible in electronic image products. Images are produced from the best available original document. 
First lasing of a high-gain harmonic-generation (HGHG) free-electron laser (FEL) was reported in refs. [1,2]. Here, the latest results characterizing the HGHG FEL output are presented. The experiment was carried out at the Accelerator Test Facillity (ATF) at Brookhaven National Laboratory in collaboration with the Advanced Photon Source at Argonne National Laboratory. A schematic of the HGHG layout is shown in Figure 1. A coherent $10.6-\mu \mathrm{m}$ seed provided by a $\mathrm{CO}_{2}$ laser interacts with the electron beam in the first (energy-modulating) undulator, which is tuned to be resonant to $10.6-\mu \mathrm{m}$. The resulting energy modulation is then converted to spatial bunching while the electron beam traverses a dispersive section (a three-dipole chicane). In the second undulator, tuned to be resonant at $5.3 \mu \mathrm{m}$, the microbunched electron beam initially emits coherent radiation and then amplifies it exponentially until saturation is achieved. We have carried out measurements of the radiation at the radiator fundamental wavelength $(5.3 \mu \mathrm{m})$, as well as at the second harmonic $(2.65 \mu \mathrm{m})$ and the third harmonic $(1.77 \mu \mathrm{m})$.

In previous work [2], the spectral distribution of the FEL output in the neighborhood of $5.3 \mu \mathrm{m}$ was characterized using a single-shot imaging technique. The bandwidth of the HGHG output was found to be $15 \mathrm{~nm}$ FWHM, much narrower than the self-amplified spontaneous emission (SASE) bandwidth. In this note, we report results of measurements of the output pulse duration using a second-harmonic intensity autocorrelator, as well as the coherence length using an interferometer.

The electron beam parameters exhibit some small daily variation about the working point. Typical parameters are $0.8 \mathrm{nC}$ charge, $6 \mathrm{ps}$ FWHM pulse length, and 5 mm-mrad normalized emittance. The repetition rate of the ATF is $1.5 \mathrm{~Hz}$, and the

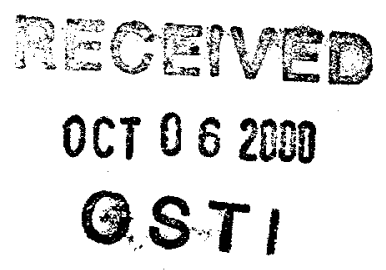


repetition rate of the $\mathrm{CO}_{2}$ laser, limited only by its pulse forming network, is once every 15 seconds. The remaining FEL parameters are given in Figure 1.

\section{Autocorrelation}

We constructed a background-free intensity autocorrelator using second-harmonic generation in a $5 \times 5 \times 1 \mathrm{~mm}^{3} \mathrm{AgGaSe}_{2}$ nonlinear crystal. The group velocity mismatch [3] and geometric beam overlap in the crystal allow better than 0.5 -ps resolution. The resolution of $1 \mathrm{ps}$ is predominantly determined by the fluctuations of the electron beam and the $\mathrm{CO}_{2}$ laser performance. The output radiation is measured with a single-element InSb photoconductive detector. The signal versus delay time (between the two arms of the autocorrelator) is shown in Figure 2. Assuming a Gaussian pulse shape, the pulse duration is found be $8.4 / \sqrt{ } 2=5.9$ ps. For an HGHG output pulse with energy $100 \mu \mathrm{J}$ and pulse length $5.9 \mathrm{ps}$, the output power is $17 \mathrm{MW}$, within a factor of two of the theoretical prediction of $35 \mathrm{MW}$.

\section{Interferometer}

An interferometer was used to investigate the temporal coherence of the HGHG output. The retroreflecting mirror in one arm was tilted off-axis and translated while the fringe contrast of the interference pattern was recorded on a thermal imaging camera, as shown in Figure 3. Note that in order to collect more light we added a cylindrical mirror to produce a line-type image on the thermal camera. The variation of fringe contrast as a function of mirror displacement, plotted in Figure 4, is a measure of the coherence length of the pulse. The optical coherence length is measured as $1.6 \mathrm{~mm}$, or $5.3 \mathrm{ps,} \mathrm{based} \mathrm{upon}$ 
the delay change. The close agreement between pulse duration and coherence length indicates nearly full longitudinal coherence.

\section{Steering}

Using a small corrector magnet located $18.4 \mathrm{~cm}$ upstream of the radiator, we studied steering effects on electron and light beam trajectories. Five cerium-doped yttrium aluminum garnet (YAG) beam position measurement screens located at regular spacing through the radiator were used to observe the electron trajectory. A thermal imaging camera $1.7 \mathrm{~m}$ downstream of the end of the radiator was used to measure the deviation of the light beam. Steering correctors were varied separately in each plane to induce a maximum electron beam offset of $1.0 \mathrm{~mm}$ (horizontal) and $0.5 \mathrm{~mm}$ (vertical) on the fifth YAG screen, situated $18 \mathrm{~cm}$ downstream of the exit of the radiator. The electron beam steering produced a maximum HGHG output centroid displacement of $2.5 \mathrm{~mm}$ (horizontal) and $1.0 \mathrm{~mm}$ (vertical) at the thermal camera.

The horizontal steering of the electron beam did not cause observable intensity reduction. Figure 5 shows the horizontal (top) and vertical (bottom) trajectories for the two cases. The measured shift of the optical HGHG output position at the thermal camera and the shift of the electron beam position on the fifth YAG screen suggest that HGHG output follows the electron beam.

When we steered the electron beam vertically by $0.5 \mathrm{~mm}$ at the fifth YAG screen, we observed about $1 \mathrm{~mm}$ displacement at the thermal camera. In this case, the intensity of the output was reduced because the radiator is a planer wiggler, i.e., the magnetic field increases as one moves out of the midplane in the vertical direction. Due to the change in 
magnetic field, the resonant wavelength shift is about $25 \mathrm{~nm}$. Since the bandwidth of the HGHG is $20 \mathrm{~nm}$, this field change causes the electrons to lose resonance with the radiation generated earlier in the wiggler.

\section{Electron Beam Modulation and Output Radiation Harmonics}

The electron beam energy modulation was determined using the electron energy spectrometer after the radiator section. In the HGHG process, energy modulation of the electron beam is generated in two ways: (1) through the initial interaction of the seed laser and electron beams in the modulator; and (2) through the HGHG FEL interaction itself in the radiator. The energy modulation produced in the radiator dominates. The electron beam images after the spectrometer (a) with $\mathrm{CO}_{2}$ laser on, and (b) with the $\mathrm{CO}_{2}$ laser off, are shown in Figure 6. With the $\mathrm{CO}_{2}$ laser on, the energy modulation is seen to be $2.5 \%$.

The amount of modulation, as well as the strength of the higher harmonics, indicates that saturation or near-saturation has been reached. The higher harmonics have been studied theoretically in refs. [4-7]. Here, we have measured the fundamental (5.3 $\mu \mathrm{m})$, second $(2.65 \mu \mathrm{m})$, and third $(1.77 \mu \mathrm{m})$ harmonics relative to the radiator as a function of electron energy modulation, using the InSb detector in conjunction with the appropriate bandpass and neutral density filters, to produce similar signal levels on the detector. The responsivity of the InSb detector is a factor of 0.67 less at $2.65 \mu \mathrm{m}$ and a factor of 0.5 less at $1.77 \mu \mathrm{m}$ as compared to the 5.3- $\mu \mathrm{m}$ responsivity. For the fundamental (5.3 $\mu \mathrm{m})$, a $10-\mathrm{nm}$ bandpass filter with $80 \%$ transmission through this band was used with $1 \times 10^{6}$ attenuation. For the second harmonic $(2.65 \mu \mathrm{m})$, a $35-\mathrm{nm}$ bandpass filter 
with $75 \%$ transmission through this band was used with $1 \times 10^{3}$ attenuation. For the third harmonic $(1.77 \mu \mathrm{m})$, a $35-\mathrm{nm}$ bandpass filter with $60 \%$ transmission through this band was used with $1 \times 10^{4}$ attenuation.

In Figure 7, we plot the harmonic contents $(\mu \mathrm{J})$ of the first three harmonics versus electron beam energy modulation (\%). The rapid increase of the harmonic intensities at $2.65 \mu \mathrm{m}$ and $1.77 \mu \mathrm{m}$ as the electron energy modulation approaches $2.5 \%$ is strong evidence of saturation. In Table 1, the theoretical predictions and experimental measurements of the ratios of the harmonic-to-fundamental energies are presented for a $2.5 \%$ electron beam energy modulation. One sees that there is good agreement between experiment and theory.

\section{Conclusion}

New results in the HGHG experiment have provided additional confirmation of theory. For the HGHG output, we used an autocorrelator to measure the intensity pulse length and an interferometer to measure the coherence length of the HGHG output. The agreement of these two measurements indicates full longitudinal coherence. Using a spectrometer, we have previously determined [2] the single-shot spectrum in the neighborhood of $5.3 \mu \mathrm{m}$ to have a $15-\mathrm{nm}$ FWHM bandwidth. The measured pulse duration of $5.9 \mathrm{ps}$ and bandwidth of $15 \mathrm{~nm}$ correspond to a time-bandwidth product that is a factor of $\sim 1.7$ larger than the minimum. However, the bandwidth and pulse duration measurement were not carried out on the same day, therefore the e-beam pulse shape might not be identical for the two measurements. To more precisely investigate the timebandwidth product, we plan to carry out the spectral and pulse duration measurements in 
a single run. The intensity of the second $(2.65 \mu \mathrm{m})$ and third $(1.77 \mu \mathrm{m})$ nonlinear harmonics relative to the radiator section resonant wavelength $(5.3 \mu \mathrm{m})$ were measured to be in reasonable agreement with theory, and their nonlinear dependence on electron energy modulation provides strong evidence of saturation. Finally, the ability to steer the HGHG output will be useful in the future as a technique to align cascaded HGHG sections in an $x$-ray FEL [8-10].

\section{References}

[1] L.-H. Yu, M. Babzien, I. Ben-Zvi, L.F. DiMauro, A. Doyuran, W. Graves, E. Johnson, S. Krinsky, R. Malone, I. Pogorelsky, J. Skaritka, G. Rakowsky, L. Solomon, X.J. Wang, M. Woodle, V. Yakimenko, S.G. Biedron, J.N. Galayda, E. Gluskin, J. Jagger, V. Sajaev, I. Vasserman, "First Lasing of a High-Gain Harmonic Generation Free-Electron Laser Experiment," Nucl. Instrum. Meth. A445 (2000) 301.

[2] L.-H. Yu, M. Babzien, I. Ben-Zvi, L.F. DiMauro, A. Doyuran, W. Graves, E. Johnson, S. Krinsky, R. Malone, I. Pogorelsky, J. Skaritka, G. Rakowsky, L. Solomon, X.J. Wang, M. Woodle, V. Yakimenko, S.G. Biedron, J.N. Galayda, E. Gluskin, J. Jagger, V. Sajaev, I. Vasserman, "High-Gain Harmonic Generation Free-Electron Laser," Science 289 (2000) 932.

[3] V.G. Dmitriev, G.G. Gurzadyan, D.N. Nikogosyan, Handbook of Nonlinear Optical Crystals, 2nd Ed. (Springer, Berlin, Heidelberg 1997).

[4] R. Bonifacio, L. De Salvo, and P. Pierini, "Large harmonic bunching in a high-gain free-electron laser," Nucl. Instr. Meth. A293 (1990) 627. 
[5] H.P. Freund, S.G. Biedron, S.V. Milton, "Nonlinear Harmonic Generation in FreeElectron Lasers," IEEE J. Quantum Electron., 36 (2000) 275.

[6] Z. Huang, K.J. Kim, "Nonlinear Harmonic Generation of Coherent Ampliciation and Self-Amplified Spontaneous Emission," these proceedings.

[7] S.G. Biedron, H.P. Freund, X.J. Wang, and L.-H. Yu, "Nonlinear Harmonics in the High-Gain Harmonic Generation (HGHG) Experiment," these proceedings.

[8] L.H. Yu , "Harmonic generation of hard X-rays," Proceedings of the IFCA Advanced Beam Dynamics Workshop on Future Light Sources, C.E. Eyberger, Ed., Argonne National Laboratory, Argonne, IL (1999), (URL: http://www. aps.anl.gov/conferences/ FLSworkshop/proceedings/papers/wg1-01.pdf).

[9] Juhao Wu, L.H. Yu, "Cascading Stages of High-Gain Harmonic Generation to Produce Coherent Hard X-rays," these proceedings.

[10] S.G. Biedron, S.V. Milton, and H.P. Freund, "Modular Approach to Achieving the Next Generation Light Source," these proceedings.

\section{LIST OF FIGURES}

Figure 1: HGHG experiment schematic and design parameters.

Figure 2: Signal versus delay after adjusting one arm of the autocorrelator. 
Figure 3: Contrast of the interference pattern on a thermal imaging camera from the interferometer.

Figure 4: Variation of fringe contrast as a function of mirror displacement revealing the coherence length of the HGHG radiation pulse.

Figure 5: Horizontal and vertical trajectory of the electron beam after steering.

Figure 6: The energy modulation of the electron beam image after the energy spectrometer (a) with and (b) without the $\mathrm{CO}_{2}$ beam present.

Figure 7: Harmonic content $(\mu)$ versus electron beam energy modulation (\%). 


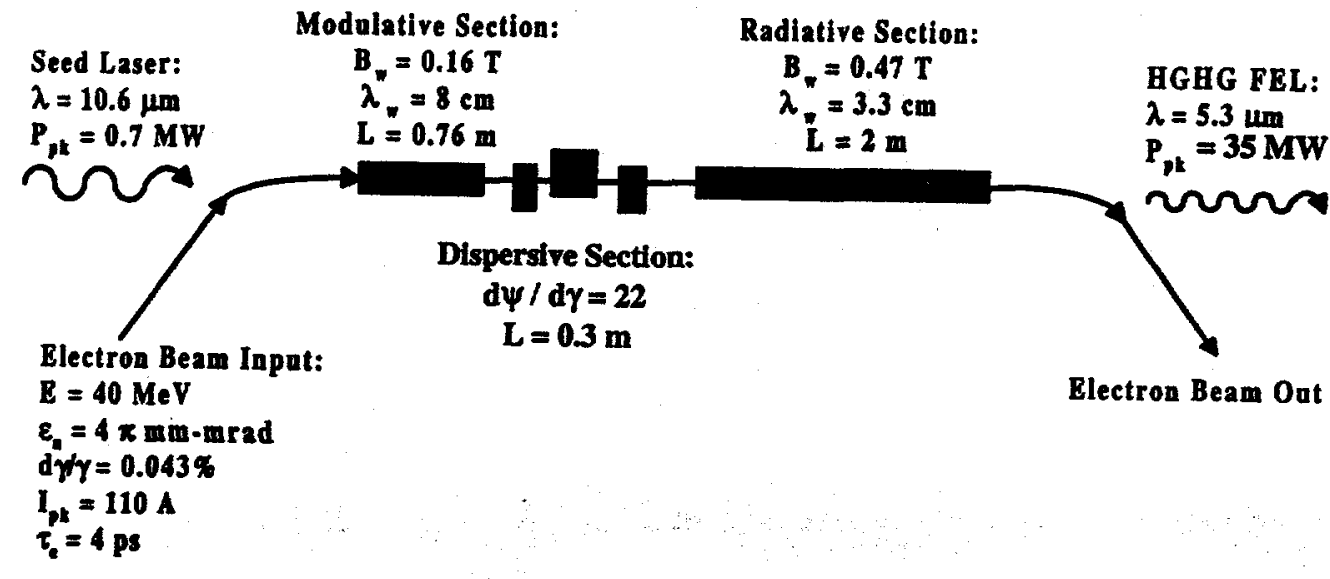

Figure 1 


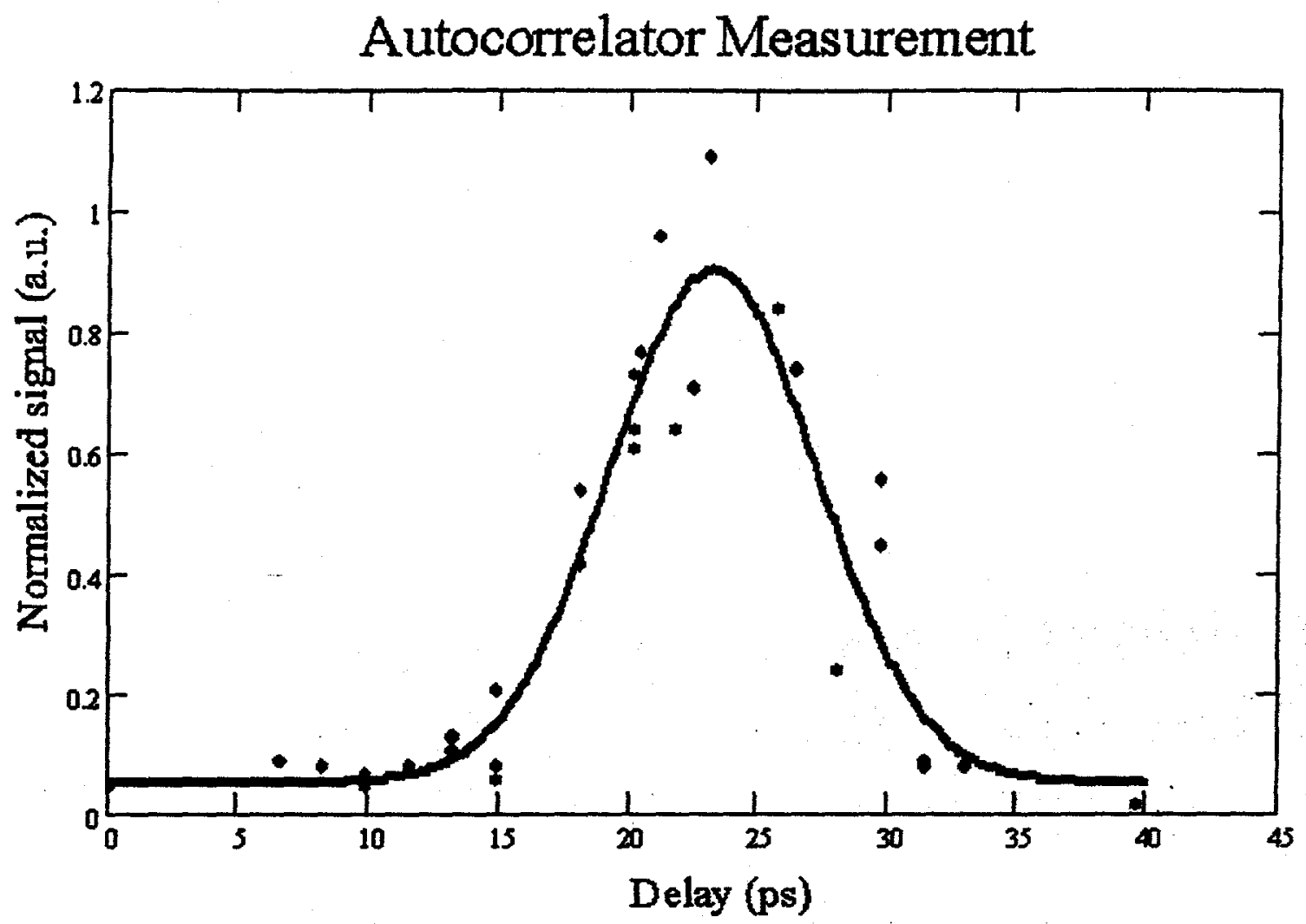

Figure 2 

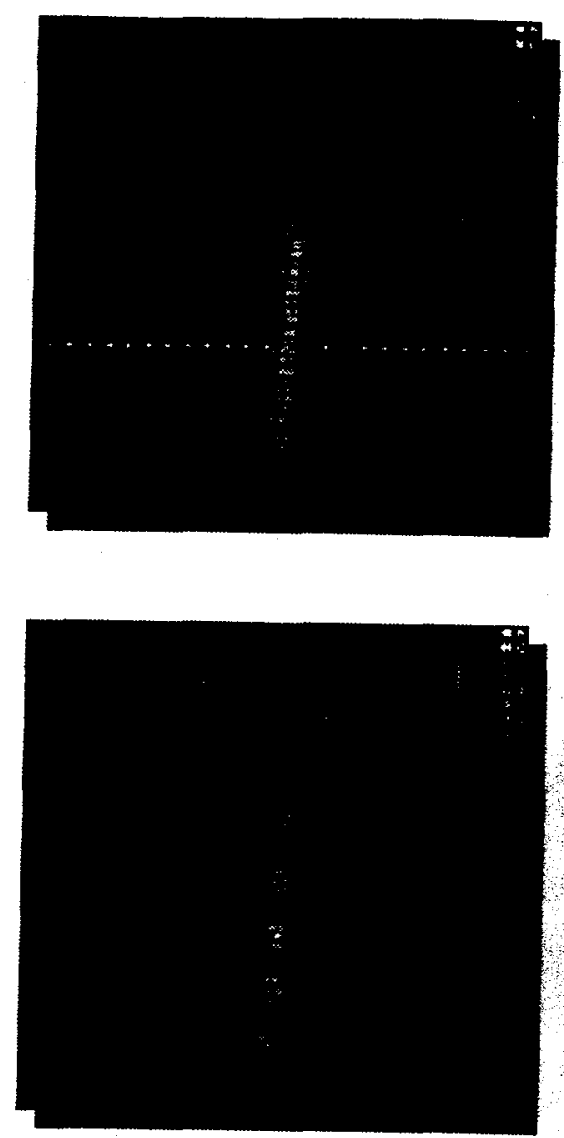

Figure 3 


\section{Interferometer Measurement}

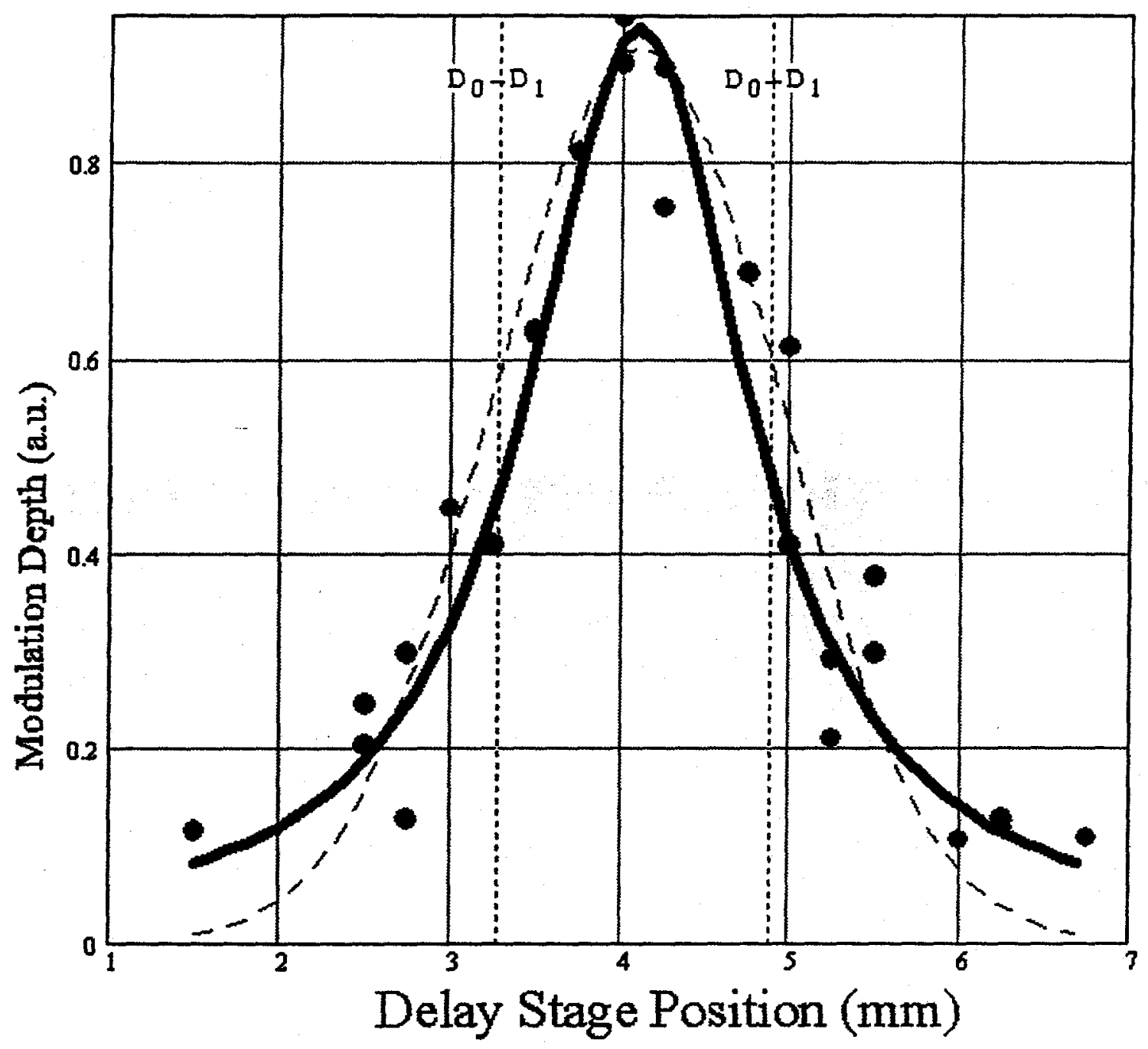

Figure 4 


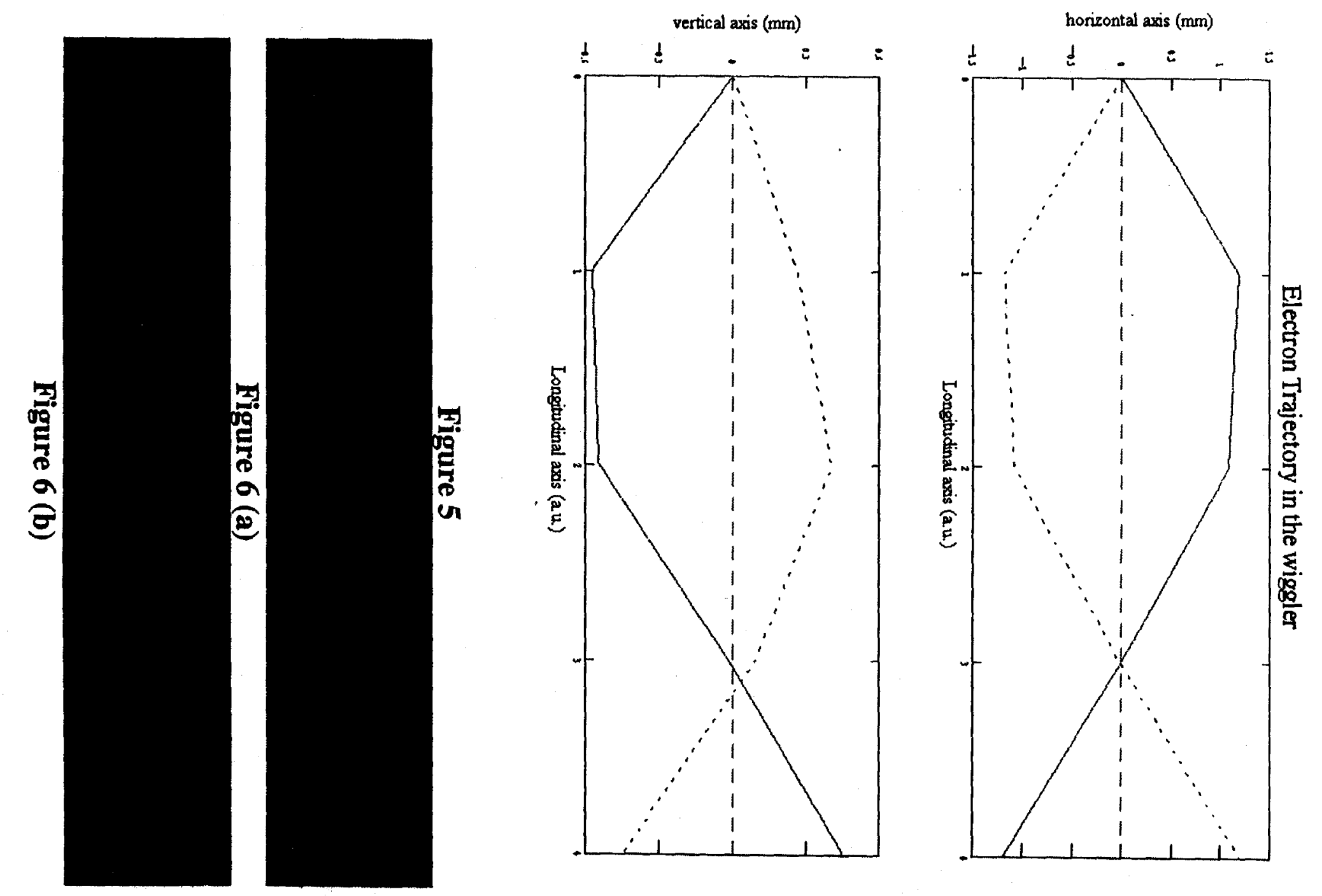




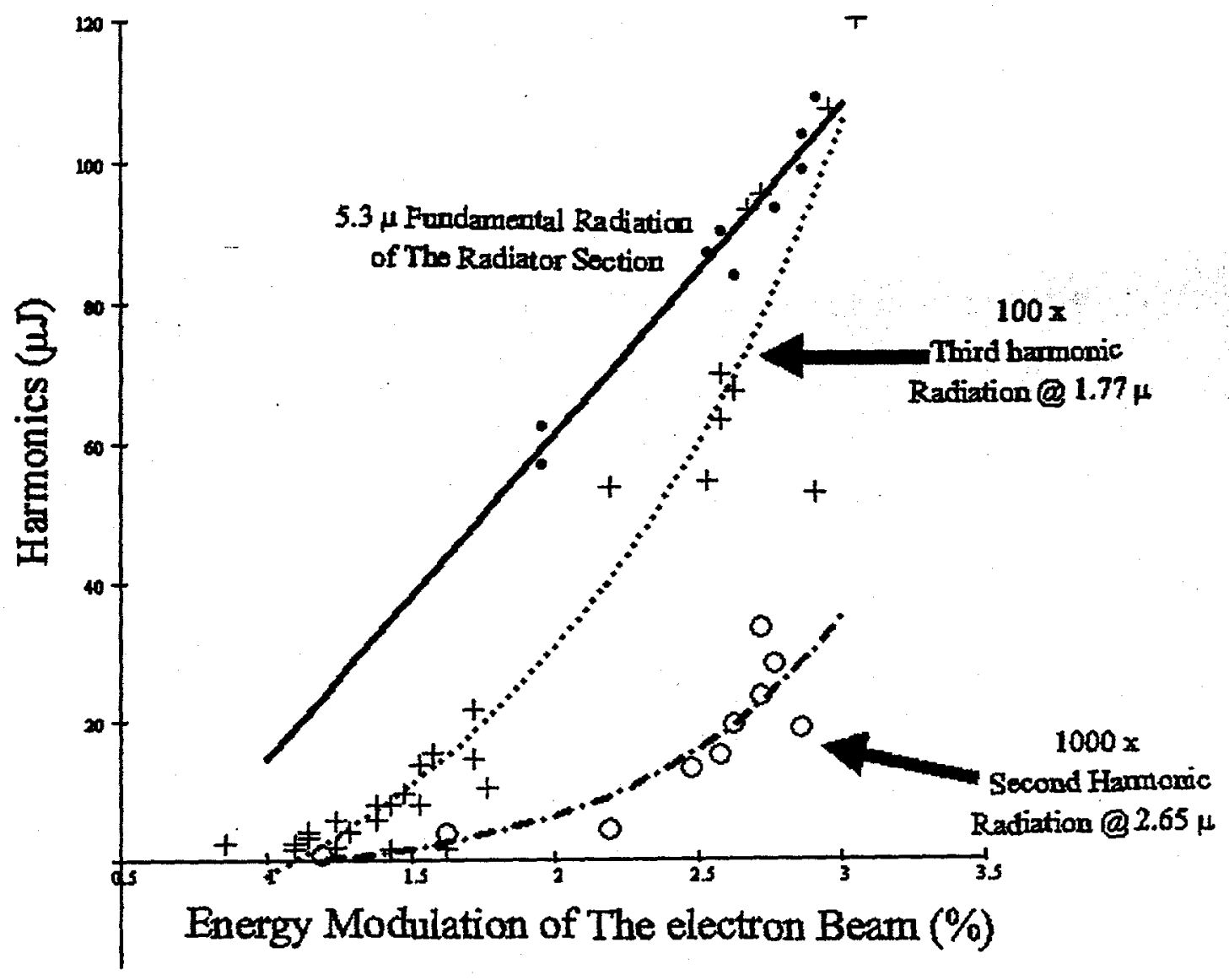

Figure 7 


\section{List of Tables}

Table 1: The theoretical and experimentally measured harmonic-to-fundamental ratios.

Table 1

Wavelength Simulation

$2.65 \mu \mathrm{m}$ $6 \times 10^{-4}$

$1.77 \mu \mathrm{m}$

$1 \times 10^{-2}$

Experiment

$2 \times 10^{-4}$

$0.8 \times 10^{-2}$ 


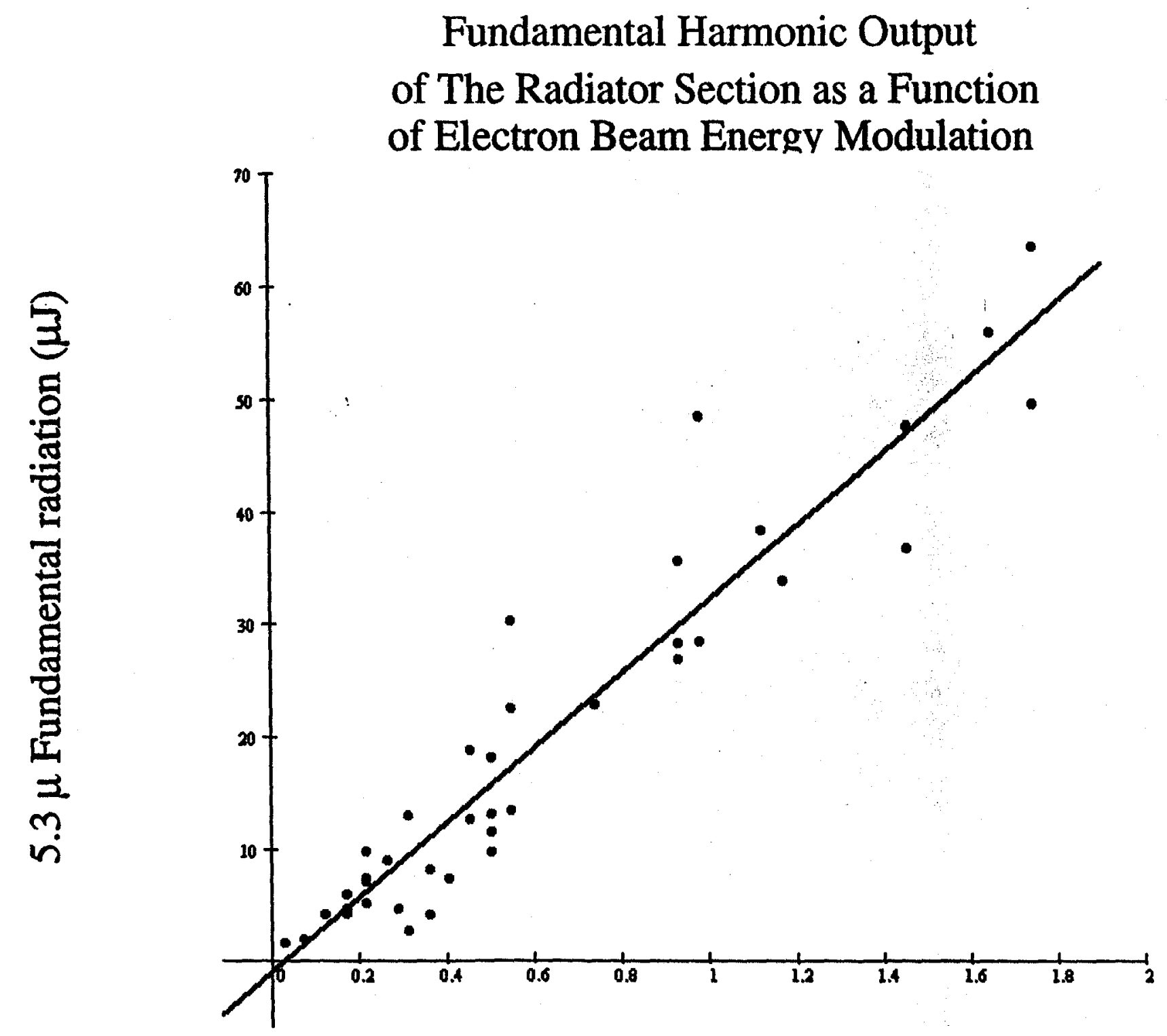

Energy Modulation of The electron Beam (\%) 


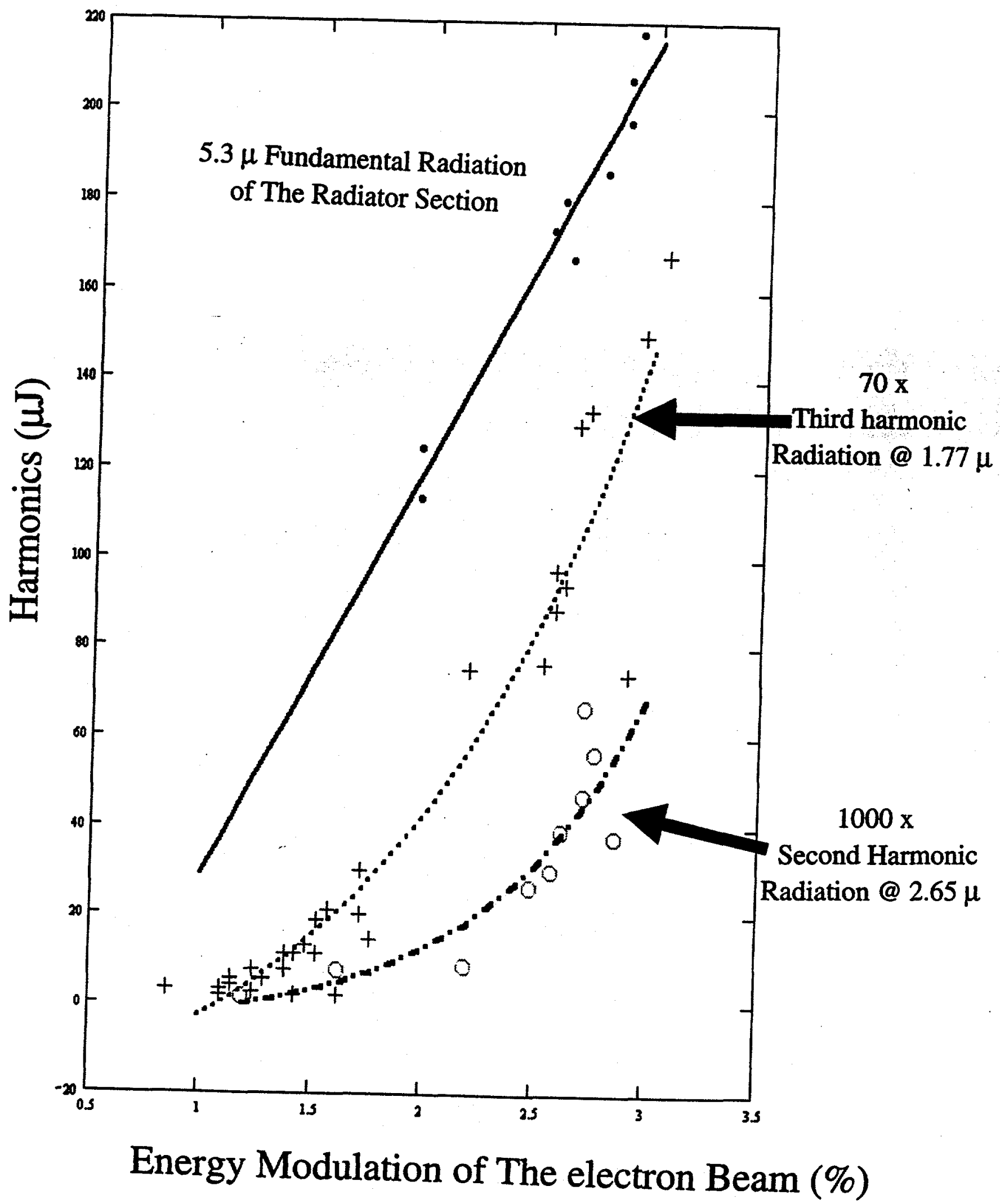




\section{For a modulation of $2.5 \%$ Harmonic/Fundamental}

\begin{tabular}{|l|l|l|l|}
\hline & simulation & $\begin{array}{l}\text { Analytical } \\
\text { Theory }\end{array}$ & Experiment \\
\hline $2^{\text {nd }}$ harmonic & $\begin{array}{l}6^{*} 10^{-4} \\
\text { Biedron et al. }\end{array}$ & $\begin{array}{l}3^{*} 10^{-4} \\
\text { Juhau Wu }\end{array}$ & $1.5^{*} 10^{-4}$ \\
\hline $3^{\text {rd } \text { harmonic }}$ & $\begin{array}{l}10^{-2} \\
\text { Biedron et al. }\end{array}$ & $\begin{array}{l}10^{-2} \\
\text { Z.R. Huang }\end{array}$ & $10^{-2}$ \\
\hline
\end{tabular}




\section{Conclusions}

- The single-shot spectrum of HGHG has been measured and it is consistent with the multi-shot spectrum measurement

- HGHG can be steered by steering the electron beam and we observe optical guiding

- The pulse length of the HGHG has been measured using two different methods

- Autocorrelator measures the intensity pulse length of the beam.

* Michelson Interferometer measures the coherence length of the beam.

Since they are in a very good agreement, pulse is fully coherent.

- Preliminary harmonic content of the HGHG has been measured and observed very good agreement with the theoretical simulations 